\title{
A STRONG PARROTT THEOREM
}

\author{
CIPRIAN FOIAS AND ALLEN TANNENBAUM
}

(Communicated by John B. Conway)

Dedicated to Professor Bela Sz.-Nagy, one of the great masters of operator theory on the occation of his seventy-fifth birthday

\begin{abstract}
In this note we discuss a strengthened version of a theorem due to Parrott [8] in operator dilation theory. We relate our result to the one-step extension procedure of Adamjan-Arov-Krein [1].
\end{abstract}

\section{INTRODUCTION}

One of the neatest results in extension, dilation, and interpolation theory is Parrott's theorem [8]. Basically, this result gives necessary and sufficient conditions for the completion of a 2 by 2 block operator-valued matrix (on a Hilbert space) to a contraction. (See Theorem 1 below for the precise formulation.) This theorem can be used as the crucial step in a proof of the Sz.-Nagy-Foias commutant lifting theorem [9], and thus can be applied to the one-step dilation procedure of Adamjan-Arov-Krein [1] for Hankel operators, and to give a proof of the Nehari theorem for the distance of an $L^{\infty}$ function to $H^{\infty}$. Parrott's theorem moreover has found a number of uses in systems theory [7].

Now an operator (when it exists) derived in completing the given 2 by 2 block operator to a contraction is in general not unique. In fact, Davis-KahanWeinberger [3] parametrize all such possible completions. (See also the relevant papers [2] and [5].) A number of interpolation problems are then concerned with picking out a special class of completions which are interesting from either the mathematical or physical point of view (or both). For example, one of the most studied completions is the so-called "maximal entropy" solution which is important both mathematically and physically, and thus has a large literature devoted to it. See for example [4], and the references therein.

Received by the editors October 25, 1988 and, in revised form, December 7, 1988.

1980 Mathematics Subject Classification (1985 Revision). Primary 47A20; Secondary 93C05.

Key words and phrases. Parrott's theorem, dilation theory, one-step extension, Hankel operator, interpolation theory.

This work was supported in part by grants from the Research Fund of Indiana University, Department of Energy DE-FG02-86ER25020, National Science Foundation ECS-8704047, DMS8811084, and by the Air Force Office of Scientific Research AFOSR-88-0020. 
In this note, we prove a strengthened version of Parrott's theorem. A very special case of this result arose in our study of the singular values and vectors of the "four block operator" from control theory and their connection to the generalization of certain interpolation results from [1]. This led us to formulate and prove Theorem 2 below in its full generality. (See [6] for a discussion of how a strengthened Parrott's theorem appears in the generalization of the Adamjan-Arov-Krein [1] interpolation-theoretic interpretation of the singular values of the Hankel to the four block operator.) Moreover, it turns out that this strong version of Parrott picks out an extension which is in a certain sense "opposite" to the maximal entropy one. (See Section 4 below.) Thus besides elucidating the spectral properties of a certain key operator from control, these extensions seem to have some intriguing mathematical properties which could also have some applied interest. We should note that our proof of the strong Parrott theorem employs the standard Parrott result in a number of steps.

We now summarize the contents of this paper. In Section 2, we state a general version of Parrott's result. In Section 3, we then prove our main theorem. In Section 4, we give the matrix version of this theorem, and apply it to the classical one-step extension procedure of Adamjan-Arov-Krein [1] in the Hankel case. Of course, this is only one possible example, and our theorem can be applied to any of the classical interpolation problems, and can even be used to prove a strong commutant lifting theorem. We plan to discuss all of this in a sequel to the present note.

\section{Preliminaries on Parrott's lemma}

In this section we would like to set up some notation, and briefly describe Parrott's theorem from [8]. Throughout this paper, by "operator" we mean "bounded linear operator" on the appropriate (separable) Hilbert space.

Let $T: H \rightarrow K$ be a contraction from the Hilbert space $H$ into the Hilbert space $K$. If $H_{0} \subset H$, and $K_{0} \subset K$ are any closed subspaces, then the operators

$$
T_{0}:=T \mid H_{0}: H_{0} \rightarrow H \quad \text { and } \quad T_{0}^{\prime}:=P_{K_{0}} T: H \rightarrow K_{0}
$$

are contractions satisfying

$$
P_{K_{0}} T_{0}=T_{0}^{\prime} \mid H_{0} .
$$

Parrott's theorem is the converse of this simple observation. More precisely, we have:

Theorem 1 (Parrott's Theorem). Let $H, K, H_{0}, K_{0}$ be as above. Then if the operators $T_{0}: H_{0} \rightarrow H, T_{0}^{\prime}: H \rightarrow K_{0}$ are any contractions satisfying (2), there exists a contraction $T: H \rightarrow K$ such that (1) holds.

We may put this into the more familiar matrix form. Let $H_{1}, H_{2}, K_{1}, K_{2}$ be Hilbert spaces. Consider the operator

$$
\left[\begin{array}{ll}
X & B \\
C & D
\end{array}\right]: H_{1} \bigoplus H_{2} \rightarrow K_{1} \bigoplus K_{2}
$$


where

$$
\max \left\{\left\|\left[\begin{array}{l}
B \\
D
\end{array}\right]\right\|,\|[C D]\|\right\} \leq 1 .
$$

Then Theorem 1 means that there exists $X: H_{1} \rightarrow K_{1}$ such that

$$
\left\|\left[\begin{array}{ll}
X & B \\
C & D
\end{array}\right]\right\| \leq 1
$$

We should note that from [3], one can parametrize all possible $X$ which satisfy (4). See also [5].

\section{The strong Parrott theorem}

We formulate and prove our main result in this section. We use the notation of Section 2 here. We begin by adding a bit more notation.

Let $V: H_{1} \rightarrow K_{1}$ be unitary, where $H_{1}$ and $K_{1}$ are closed subspaces of the Hilbert spaces $H$ and $K$ respectively. Assume that the contraction $T: H \rightarrow K$ (see Section 2) satisfies

$$
T \mid H_{1}=V .
$$

Then clearly from (1) we have that

$$
P_{K_{0}} V=T_{0}^{\prime} \mid H_{1}
$$

Now since

it follows that

$$
\left\|T h_{1}\right\|=\left\|V h_{1}\right\|=\left\|h_{1}\right\|, \quad \forall h_{1} \in H_{1},
$$

$$
V^{*} V h_{1}=h_{1}=T^{*} T h_{1}=T^{*} V h_{1}, \quad \forall h_{1} \in H_{1},
$$

and so we see that

$$
T^{*} \mid K_{1}=V^{*} .
$$

But $T_{0}=T \mid H_{0}$ implies that $P_{H_{0}} T^{*}=T_{0}^{*}$, and so

$$
T_{0}^{*} \mid K_{1}=P_{H_{0}} V^{*}
$$

or equivalently

$$
V P_{H_{1}} \mid H_{0}=P_{K_{1}} T_{0} \text {. }
$$

The strong version of Parrott's theorem is the converse of this set-up. More precisely, we have

Theorem 2. Notation and hypotheses as in Theorem 1. (Thus we have in particular that the operators $T_{0}: H_{0} \rightarrow H$ and $T_{0}^{\prime}: H \rightarrow K_{0}$ are contractions and satisfy (2).) Suppose moreover that $V: H_{1} \rightarrow K_{1}$ is unitary and satisfies (6) and (9). Then there exists a contraction $T: H \rightarrow K$ such that (1) and (5) hold. Proof. Define $H_{2}:=\overline{H_{1}+H_{0}}$ and $K_{2}:=\overline{K_{1}+K_{0}}$. From Parrott's theorem, there exists a contraction $T^{\prime}: H_{2} \rightarrow K$ such that

$$
T^{\prime} \mid H_{1}=V \text { and } P_{K_{0}} T^{\prime}=T_{0}^{\prime} \mid H_{2} \text {. }
$$


(Note that (6) is precisely (2) with $T_{0}$ and $H_{0}$ replaced by $V$ and $H_{1}$.) Now define $T_{2}^{\prime}:=P_{K_{2}} T^{\prime}$. Then for $h_{1} \in H_{1}$, we have

$$
T_{2}^{\prime} h_{1}=P_{K_{2}} T^{\prime} h_{1}=P_{K_{2}} V h_{1}=V h_{1},
$$

and for any $h_{0} \in H_{0}$, using (2), we have

$$
P_{K_{0}} T_{2}^{\prime} h_{0}=P_{K_{0}} T^{\prime} h_{0}=T_{0}^{\prime} h_{0}=P_{K_{0}} T_{0} h_{0} .
$$

Moreover, clearly

$$
P_{K_{1}} T_{2}^{\prime} h_{0}=P_{K_{1}} T^{\prime} h_{0} .
$$

Next since $\left\|T^{\prime}\right\| \leq 1$ and $T^{\prime} \mid H_{1}=V$ is an isometry, we see for $h \in H_{2} \ominus H_{1}$, $h_{1} \in H_{1}$ that

$$
\left\langle T^{\prime} h, T^{\prime} h_{1}\right\rangle=\left\langle h, T^{\prime *} T^{\prime} h_{1}\right\rangle=\left\langle h, h_{1}\right\rangle=0
$$

so that $T^{\prime}\left(H_{2} \ominus H_{1}\right) \subset K \ominus K_{1}$. It therefore follows

$$
P_{K_{1}} T^{\prime}=P_{K_{1}} T^{\prime} P_{H_{1}}=V P_{H_{1}},
$$

and so using (13) and (9), we get that

$$
P_{K_{1}} T_{2}^{\prime} h_{0}=P_{K_{1}} T_{0} h_{0} \quad\left(h_{0} \in H_{0}\right) .
$$

But (12) and (14) imply

$$
T_{2}^{\prime} \mid H_{0}=P_{K_{2}} T_{0} .
$$

Next note that Equation (15) is precisely (2) with $K_{0}$ and $T_{0}^{\prime}$ replaced by $K_{2}$ and $T_{2}^{\prime}$. Hence from Parrott's theorem, there exists a contraction $T_{2}: H_{2} \rightarrow K$ satisfying

$$
T_{2} \mid H_{0}=T_{0}, \quad P_{K_{2}} T_{2}=T_{2}^{\prime} .
$$

Now for any $h_{1} \in H_{1}$, we have by (11) that

$$
\left\|T_{2}^{\prime} h_{1}\right\|=\left\|V h_{1}\right\|=\left\|h_{1}\right\| \geq\left\|P_{K_{2}} T_{2} h_{1}\right\|=\left\|T_{2}^{\prime} h_{1}\right\|
$$

and thus

$$
T_{2} h_{1}=P_{K_{2}} T_{2} h_{1}=T_{2}^{\prime} h_{1}=V h_{1},
$$

which means that

$$
T_{2} \mid H_{1}=V .
$$

Next notice that $P_{K_{0}} T_{2}=P_{K_{0}} T_{2}^{\prime}$, and that

$$
\begin{array}{lll}
P_{K_{0}} T_{2}^{\prime} h_{1}=P_{K_{0}} V h_{1}=T_{0}^{\prime} h_{1}, & \forall h_{1} \in H_{1}, & \text { by }(11),(6) . \\
P_{K_{0}} T_{2}^{\prime} h_{0}=P_{K_{0}} T_{0} h_{0}=T_{0}^{\prime} h_{0}, & \forall h_{0} \in H_{0}, & \text { by (12), (2). }
\end{array}
$$

It follows that

$$
P_{K_{0}} T_{2}=T_{0}^{\prime} H_{2}
$$


This is once again (2) with $H_{0}$ and $T_{0}$ replaced by $H_{2}$ and $T_{2}$. Applying therefore Parrott's theorem as above, we obtain a contraction $T: H \rightarrow K$ such that

$$
T \mid H_{2}=T_{2} \quad \text { and } \quad P_{K_{0}} T=T_{0}^{\prime} .
$$

By virtue of (16), (17), and (19), $T$ has all the required properties, which completes the proof.

Remarks. Equations (16), (17) show that $T_{2}$ is uniquely determined by $T_{0}$ and $V$. Thus the freedom in choosing $T$ is the same as that of the Parrott theorem when applied to $T_{2}$ and $T_{0}^{\prime}$. Hence from the Davis-Kahan-Weinberger parametrization [3], it follows that all solutions $T$ of the extension problem formulated in Theorem 2, can be parametrized by the set of contractions $R: D_{1} \rightarrow D_{2}$, where $D_{1}$ and $D_{2}$ are certain defect spaces associated to the given operators. (See [3] and [5] for the precise formulae.) Consequently, the extension $T$ is unique if and only if at least one of the $D_{i}=\{0\}$ for $i=1,2$.

\section{MAtrix formulation of the strong Parrott theorem}

In this section, we would like to give the matrix formulation of Theorem 2, and moreover apply it to the classical one-step extension procedure of AdamjanArov-Krein [1].

As in Section 2, let $H_{1}, H_{2}, K_{1}, K_{2}$ be Hilbert spaces. Consider the operator

$$
\left[\begin{array}{ll}
X & B \\
C & D
\end{array}\right]: H_{1} \bigoplus H_{2} \rightarrow K_{1} \bigoplus K_{2}
$$

where

$$
\max \left\{\left\|\left[\begin{array}{l}
B \\
D
\end{array}\right]\right\|,\left\|\left[\begin{array}{ll}
C & D
\end{array}\right]\right\|\right\} \leq 1 .
$$

Let

$$
\left[\begin{array}{l}
Y_{1} \\
Y_{2}
\end{array}\right]: H \rightarrow H_{1} \bigoplus H_{2}, \quad\left[\begin{array}{l}
Z_{1} \\
Z_{2}
\end{array}\right]: H \rightarrow K_{1} \bigoplus K_{2}
$$

be operators such that

$$
Y_{1}^{*} Y_{1}+Y_{2}^{*} Y_{2}=Z_{1}^{*} Z_{1}+Z_{2}^{*} Z_{2}
$$

Then the strong Parrott theorem (Theorem 2) means that there exists an $X: H_{1} \rightarrow K_{1}$ such that

$$
\left\|\left[\begin{array}{ll}
X & B \\
C & D
\end{array}\right]\right\| \leq 1, \quad\left[\begin{array}{ll}
X & B \\
C & D
\end{array}\right]\left[\begin{array}{l}
Y_{1} \\
Y_{2}
\end{array}\right]=\left[\begin{array}{l}
Z_{1} \\
Z_{2}
\end{array}\right]
$$

if and only if

$$
\begin{aligned}
C Y_{1}+D Y_{2} & =Z_{2}, \\
B^{*} Z_{1}+D^{*} Z_{2} & =Y_{2} .
\end{aligned}
$$

This is probably the formulation of Theorem 2 which is the easiest to apply. 
Example. Let us apply now the matrix formulation of Theorem 2 to the one-step extension of Adamjan-Arov-Krein [1]. Consider the Hankel matrix

$$
Q=\left[\begin{array}{cccc}
x & a_{1} & a_{2} & \cdots \\
a_{1} & a_{1} & \cdot & \cdots \\
a_{2} & \cdot & \cdot & \cdots \\
\cdot & \cdot & \cdot & \cdots
\end{array}\right]
$$

and the vectors

$$
\mathbf{y}:=\left[\begin{array}{c}
y_{0} \\
y_{1} \\
\vdots \\
y_{n} \\
\vdots
\end{array}\right], \quad \mathbf{z}:=\left[\begin{array}{c}
z_{0} \\
z_{1} \\
\vdots \\
z_{n} \\
\vdots
\end{array}\right]
$$

with $\|\mathbf{y}\|=\|\mathbf{z}\|$. Then by the strong Parrott theorem, there exists an $x$ which makes $Q$ a contraction and such that $Q \mathbf{y}=\mathbf{z}$ if and only if

$$
Q_{1}:=\left[\begin{array}{cccc}
a_{1} & a_{2} & a_{3} & \cdots \\
a_{2} & a_{3} & \cdot & \cdots \\
a_{3} & \cdot & \cdot & \cdots \\
\cdot & \cdot & \cdot & \cdots
\end{array}\right]
$$

is a contraction, and

$$
Q_{1} \mathbf{y}=\left[\begin{array}{c}
z_{1} \\
z_{2} \\
\vdots \\
z_{n} \\
\vdots
\end{array}\right], \quad Q_{1}^{*} \mathbf{z}=\left[\begin{array}{c}
y_{1} \\
y_{2} \\
\vdots \\
y_{n} \\
\vdots
\end{array}\right]
$$

Let us see now in what sense the strong Parrott theorem gives a minimal entropy extension in this Hankel framework. First, assuming conditions (22), let $a_{0}$ be the $x$ in (21) such that $Q \mathbf{y}=\mathbf{z}$ with $\|Q\|=1$. By the standard Parrott theorem, there exist (unique) $a_{-j}, j \geq 1$ such that $\left\|Q_{c}\right\|=\|Q\|=1$ where

$$
Q_{c}:=\left[\begin{array}{cccc}
\vdots & \vdots & \vdots & \vdots \\
a_{-2} & a_{-1} & a_{0} & \ldots \\
a_{-1} & a_{0} & a_{1} & \ldots \\
a_{0} & a_{1} & a_{2} & \ldots \\
a_{1} & a_{2} & a_{3} & \ldots \\
\cdot & \cdot & \cdot & \ldots
\end{array}\right] .
$$

Now set

$$
f(\zeta):=\sum_{j=-\infty}^{\infty} a_{j} \zeta^{-j}, \quad \mathbf{y}(\zeta):=\sum_{j=0}^{\infty} y_{j} \zeta^{j}, \quad \mathbf{z}(\zeta):=\sum_{j=0}^{\infty} z_{j} \zeta^{j}
$$

with $\zeta \in \partial D$ (the unit circle). Denote by $H^{2}$ the standard Hardy space defined on the unit disc $D$, and $L^{2}$ the standard Lebesgue space with norm \|\|$_{2}$. Let 
$J: L^{2} \rightarrow L^{2}$ be the "flip operator" given by $J g(\zeta):=g\left(\zeta^{-1}\right)$ for $g \in L^{2}$. Then the Hankel operator associated to $f$ is defined by

$$
H_{f}:=P J M_{f} \mid H^{2}, \quad H_{f}: H^{2} \rightarrow H^{2},
$$

where $M_{f}: L^{2} \rightarrow L^{2}$ denotes multiplication by $f$, and $P: L^{2} \rightarrow H^{2}$ is orthogonal projection. From the above discussion, we see that

$$
H_{f} \mathbf{y}(\zeta)=\mathbf{z}(\zeta), \quad\left\|H_{f}\right\|=1
$$

We now claim that

$$
f(\zeta)=\frac{\mathbf{z}\left(\zeta^{-1}\right)}{\mathbf{y}(\zeta)}
$$

Indeed, we clearly have that

$$
\|\mathbf{y}(\zeta)\|_{2}=\|\mathbf{z}(\zeta)\|_{2}=\left\|H_{f} \mathbf{y}(\zeta)\right\|_{2} \leq\|f \mathbf{y}(\zeta)\|_{2} \leq\|f\|_{\infty}\|\mathbf{y}(\zeta)\|_{2} \leq\|\mathbf{y}(\zeta)\|_{2} .
$$

Hence all the inequalities in (23) are equalities, which implies that

$$
\mathbf{z}(\zeta)=P J M_{f} \mathbf{y}(\zeta)=J M_{f} \mathbf{y}(\zeta),
$$

from which the claim follows. Consequently, $f$ is unimodular, and minimizes the entropy integral [4]

$$
\frac{1}{2 \pi} \int_{0}^{2 \pi} \log \left(1-\left|f\left(e^{i \theta}\right)\right|^{2}\right) d \theta
$$

This gives the required application of the strong Parrott theorem to one-step extensions of Hankel operators. Of course, we may apply Theorem 2 to any of the classical extension problems and get a corresponding minimal entropy solution.

\section{REFERENCES}

1. V. M. Adamjan, D. Z. Arov, and M. G. Krein, Analytic properties of Schmidt pairs for a Hankel operator and the generalized Schur-Takagi problem, Math. USSR Sbornik 15 (1971), 31-73.

2. C. Davis, A factorization of an arbitrary $m \times n$ contractive operator matrix, in Toeplitz centenial, edited by Israel Gohberg, Operator Theory: Advances and Applications 4 (1982), Birkhauser, 217-232.

3. C. Davis, W. M. Kahan, and H. F. Weinberger, Norm-preserving dilations and their applications to optimal error bounds, SIAM J. Numerical Anal. 19 (1982), 445-469.

4. H. Dym and I. Gohberg, A maximum entropy principle for contractive interpolants, J. Functional Analysis 65 (1986), 83-125.

5. C. Foias and A. Frazho, Redheffer products and lifting contractions on Hilbert space, J. Operator Theory 11 (1984), 193-196.

6. C. Foias and A. Tannenbaum, On the singular values of the four block operator and certain generalized interpolation problems, invited paper for a volume dedicated to Professor Mischa Cotlar on his seventy-fifth birthday, 1988.

7. B. A. Francis, $A$ course in $\mathscr{H}_{\infty}$ control theory, Lecture Notes in Control and Information Sciences, vol. 88, Springer-Verlag, 1987. 
8. S. Parrott, On the quotient norm and the Sz. Nagy-Foias lifting theorem, J. Functional Analysis 30 (1978), 311-328.

9. B. Sz.-Nagy and C. Foias, Harmonic analysis of operators on Hilbert space, North-Holland, 1970.

Department of Mathematics, Indiana University, Bloomington, Indiana, 47405

Department of Electrical Engineering, University of Minnesota, Minneapolis, MinNeSOTA 55455 